\title{
A Multidisciplinary Approach to Treat A Subgingivally Fractured Incisor -A Case Report
}

\author{
Dr.Shristhi Sharma ${ }^{1}$,Dr.Anant Raghav Sharma ${ }^{2}$,Dr.Shubhprabhat Gupta ${ }^{3}$, \\ Dr.Shanti Prasad Maurya ${ }^{4}$, \\ (Mds Endodontics, Mba, Senior Lecturer, Dept. Of Conservative Dentistry And Endodontics, Pacific Dental \\ College And Research Centre,Bhillokabedla,Udaipur, India) \\ (Mds Periodontology, Reader, Dept. Of Periodontology, Pacific Dental College, Debari,Udaipur, India) \\ (Senior Lecturer, Dept. Of Conservative Dentistry And Endodontics, Pacific Dental College, \\ Debari, Udaipur,India) \\ (Mds Endodontics, Senior Resident, Dept. Of Dentistry, J L N Medical College And Hospital, Ajmer, India)
}

\begin{abstract}
Movement of a tooth by extrusion involves applying traction forces in all regions of the periodontal ligament to stimulate marginal apposition of crestal bone. Because the gingival tissue is attached to the root by connective tissue, the gingiva follows the vertical movement of the root during the extrusion process. Similarly, the alveolus is attached to the root by the periodontal ligament and is in turn pulled along by the movement of the root.Forced eruption may serve as an alternative treatment modality since its introduction in 1973. A 25 year old boy came with obliquely fractured maxillary left central incisor at the cervical level. The diagnosis of complicated crown fracture with apical periodontitis in relation to 21 was made. Root canal treatment of the tooth was done followed by $J$ hook cementation for the forced eruption of the tooth by orthodontic wires. After 21 days, restoration of tooth with post and core followed by crown was done.
\end{abstract}

Keywords: orthodontic extrusion, J hook, crown lengthening, cast post.

\section{Case Report}

A 25 year old man referred to the department of endodontics, 1 year after he met with a road traffic accident. His anterior teeth and upper lip were traumatized. He had attended the emergency unit of a local hospital.His medical and family history was non contributory. The intraoral examination revealed maxillary left central incisor was fractured obliquely at the cervical level. Patient was experiencing pain which was throbbing and continuous in nature since 1 week. Palatal tooth fragment was mobile and labial fragment was intact (fig.1).

\section{Radiographic Finding:-}

A periapical radiograph of tooth 11 and 21 revealed that the 21 had sustained an oblique incisal fracture involving enamel, dentin and pulp. Fracture was at the cervical level and tooth was intruded with diminished periodontal space around the root. There was no periapical pathosis or root fractures observed in relation to 21 . Only $1.5 \mathrm{~mm}$ of crown structure was clinically visible. Tooth 21 was tender on percussion and widening of PDL space could be appreciated. (fig.1).The diagnosis of complicated crown fracture with apical periodontitis in relation to 21 was made.

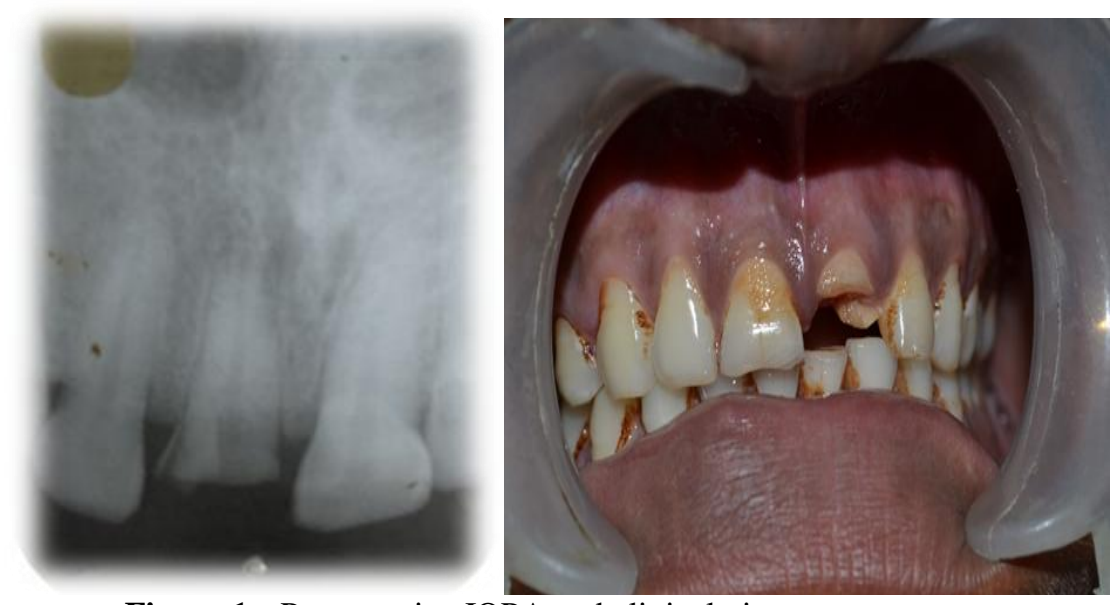

Figure 1:- Preoperative IOPA and clinical picture 
When the crown structure is less for executing any kind of prosthetic treatment there are two treatment alternatives which exist for such clinical situations: one is a surgical crown lengthening procedure, and the other is forced eruption combined with fibrotomy. In our case clinical crown structure was only $1.5 \mathrm{~mm}$ labially and 1 mm palatally.

As a surgical crown lengthening procedure compromises the supporting bone and elongates clinical crown of the adjacent teeth, the selected treatment plan was to extrude the tooth orthodontically combined with supracrestalfibrotomy. The root length inside the alveolar bone was sufficient enough to allow extrusion without compromising the periodontal support of the tooth. LA with adrenaline was administered after sensitivity testing. Fractured palatal fragment was removed with the help of forceps. The tooth was isolated under rubber dam (Hygienic Dental Dam, ColténeWhaledent, Germany). Adequate endodontic access cavity was prepared and working length radiograph was taken after initial identification of canal with \# 15 K-files (Kerr Manufacturing Co., Romulus, MI).

Root length was $20 \mathrm{~mm}$. Cleaning and shaping of the root canal was performed by using $\mathrm{K}$ file with step back technique (till $60 \mathrm{~K}$ file), canal was shaped coronally using GG drills. Copious irrigation with saline, $5.25 \%$ sodium hypochlorite solution (Dentpro, Chandigarh, India) and 17\% Ethylenediaminetetraacetic acid (EDTA) (Glyde File Prep, Densply, France) was done and $\mathrm{CaOH}$ dressing was given. In the next visit, after 7 days, master cone radiograph was taken. Before obturation, the canal was finally rinsed with $\mathrm{CHX}$, dried and sectional obturation was performed using warm verticle compaction of gutta-percha using AH Plus resin sealer (Maillefer, Dentsply, Germany).

In the third appointment, a J shaped 0.036 inch S.S. wire was cemented on the tooth 21 with GIC Type I (Fig 2). It was allowed to set and for anchorage support for extrusion, preadjusted brackets were cemented on teeth 15, 14, 13, 12, 11, 21, 22, 23, 24 and 25 for controlled extrusion. A 17 X 25 rectangular stainless steel wire was inserted into the brackets. An auxiliary 0.014 superelasticNiTi wire was overlaid on the stainless steel wire. It was tied with the lingual button using a ligature wire. A force of 40-50 gram was applied and extrusion was achieved in 4 weeks. The patient was seen every week to monitor the progress. The final crown height obtained labially and palatally was approximately $3 \mathrm{~mm}$ which was adequate for a crown ferrule and retention of the prosthesis. After active tooth movement was complete, after 21 days, the tooth was stabilized with fiber splint and supracrestalfibrotomy was done under LA. (Fig 3 and 4).

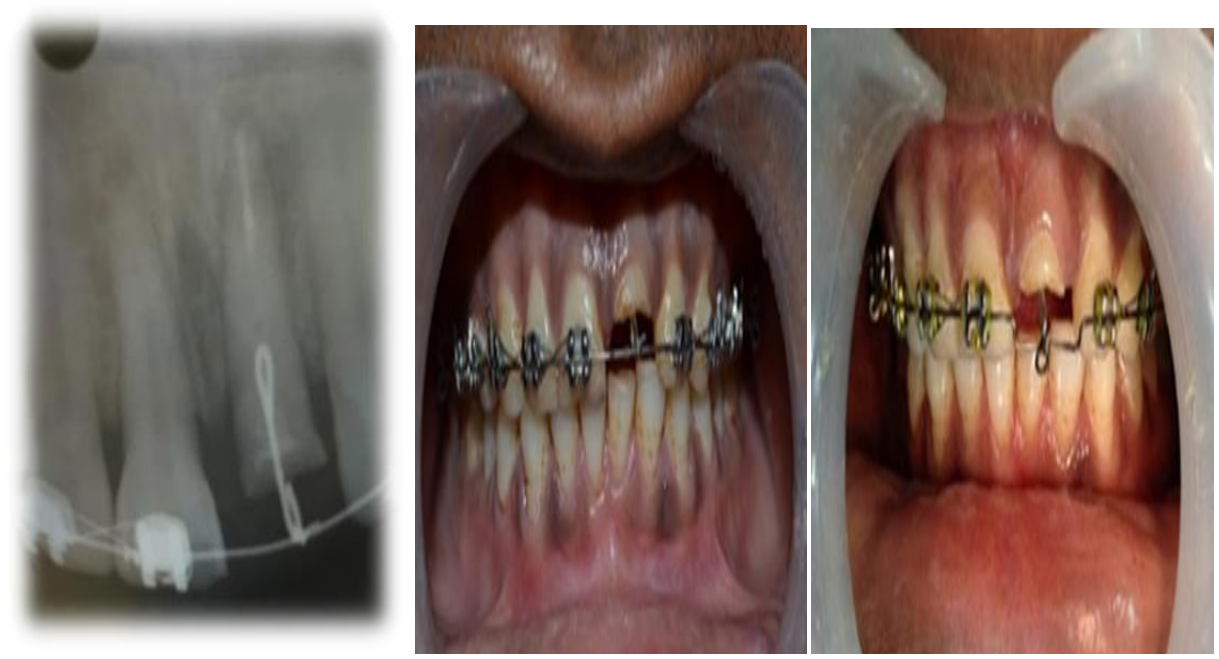

Figure 2:- Obturation IOPA and insetion of J Hook into the canal

Figure 3:- Placement of orthodontic brackets

Figure 4: After orthodontic extrusion (21 days later)

At the following appointment, restoration of tooth with post and core and crown was planned. The root canal anatomy of tooth 21 did not permit use of a conventional prefabricated glass fiber post, as the canal was oval shaped and also inclination of the tooth had to be changed. A cast gold post and core was the choice made for this situation (Fig 5). 

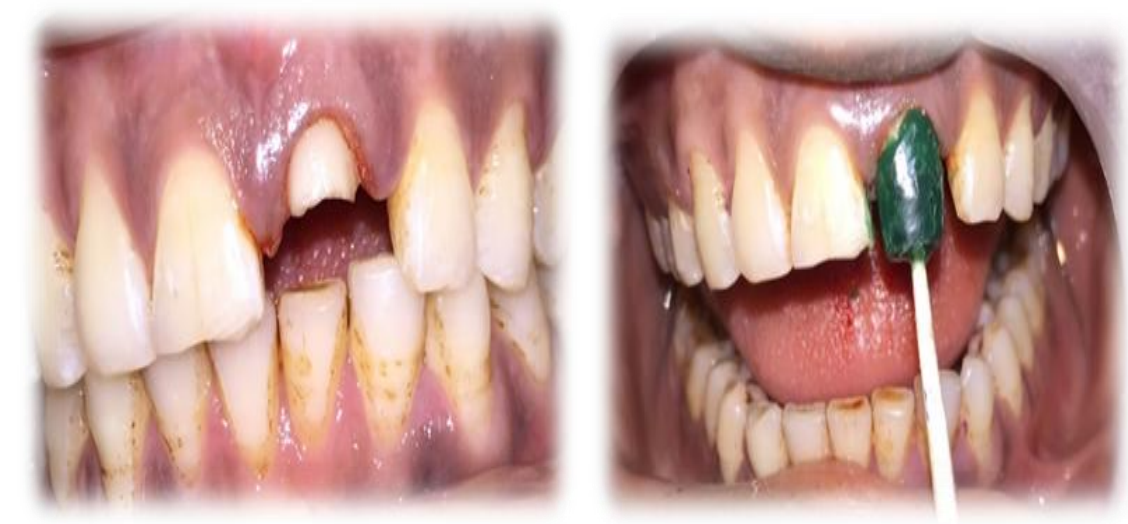

Figure 5:- Tooth preparationFigure 6:- Wax pattern
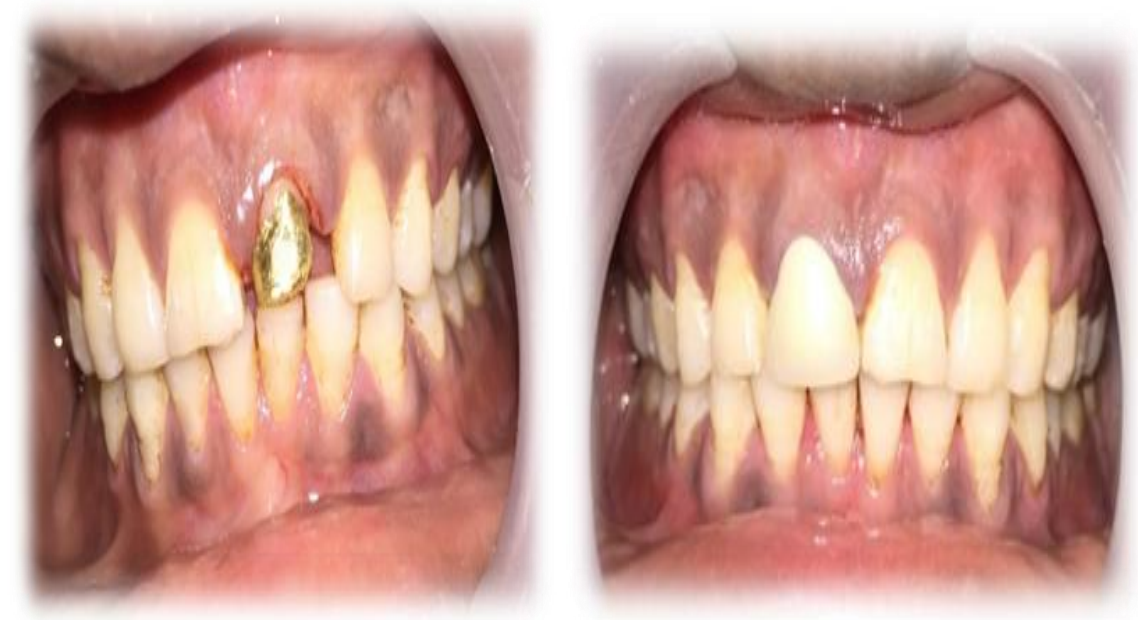

Figure 7:- Cementation of cast postFigure 8:- Cementation of PFM Crown

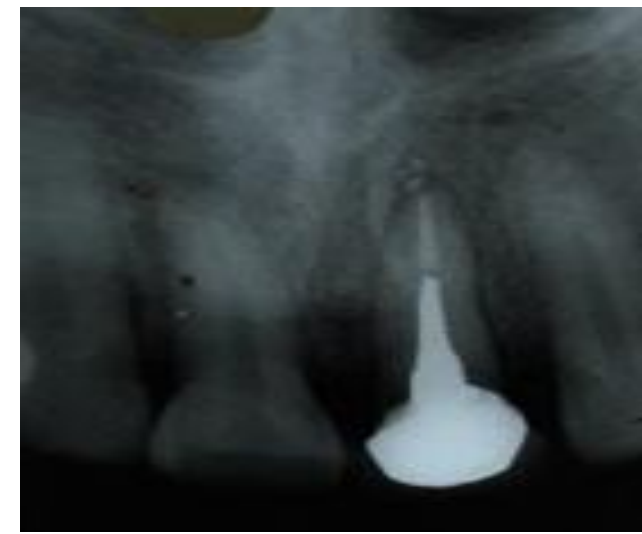

Figure 9:- Cementation IOPA

Direct wax pattern was taken with help of wax and wooden sprue. Casting of the cast post pattern was done in cast gold alloy and the cementation procedure was then performed using a type I GIC (GC Corporation) according to manufacturer's instructions (Fig 6). After final preparation of the tooth, an impression was taken using addition silicone impression material using single step putty wash technique. Shade selection of the crown was done using Vita shade guide. PFM crown was fabricated and the cementation was performed using type I GIC (Fig 7 and 8).

The patient was recalled after twelve months. The patient was asymptomatic and was satisfied with treatment outcome. A periapical radiograph demonstrated that post and core remained well adapted to post space and there was a complete healing of periapical lesion (Fig 9). 


\section{Discussion}

Forced orthodontic eruption, also known as orthodontic extrusive remodelling, forced eruption, or orthodontic extraction, is an osteophysiologic and orthodontic-based technique. The concept of the forced eruption is to raise the defect or the fractured root surface above the alveolar bone with a horizontal component of wire attached to adjacent teeth, from which a vertical force is then exerted on the root. The tooth is then held in this position until the socket heals. The tooth can then be restored on a routine basis with the crown margin on sound tooth structure. This case report highlights the use of forced orthodontic extrusion and cast gold post for rehabilitation of grossly fractured crown. Forced orthodontic extrusion was selected as it maintains acceptable crown-root ratio, biological width, good periodontal health and aesthetic demand. Other procedures like surgical crown lengthening and osteotomy have compromised aesthetic demand, inverse crown-root ratio, compromised function of adjacent teeth and the biological width.

We report a case of the multidisciplinary approach between the orthodontist, endodontist and periodontist to extrude and esthetically restore a tooth with complicated oblique crown fracture and intrusion. Teeth that were fractured and intruded which were extracted earlier, can now be saved and restored routinely, leading to a functional and esthetically pleasing result. The key to success are the right indications for the treatment and the dedication of the dentist to reassure and motivate the patient throughout the course of treatment. Orthodontic extrusion remains an accessible technique for practitioners and a beneficial technique for the patient who wishes to keep a tooth.

\section{References}

[1]. Linde J. Textbook of Clinical Periodontology. Copenhagen, Denmark: Munksgaard; 1983.

[2]. Orthodontic Extrusion as an Aid in Oral Rehabilitation Pedro Marcelo Tondelli, FabianaAkemy Kay, Marcos RikioKuabara OHDM Vol. 13 - No. 2 - June, 2014.

[3]. Normand Bach, Orthodontic Extrusion: Periodontal Considerations and Applications, J Can Dent Assoc 2004; 70(11):775-80.

[4]. Heithersay GS, Moule AJ. Anterior subgingival fractures: a review of treatment alternatives. Aust Dent J 1982; 27(6):368-76.

[5]. Berglundh T, Marinello CP, Lindhe J, Thilander B, Liljenber B. Periodontal tissue reactions to orthodontic extrusion. An experimental study in the dog. J ClinPeriodontol 1991; 18(5):330-6.

[6]. Rahul Kumar, Forced orthodontic extrusion and use of CAD/CAM for reconstruction of grossly destructed crown:A multidisciplinary approach, Journal of Conservative Dentistry | Apr-Jun 2012 | Vol 15 |Issue2.

[7]. AnupamaSwarnkar A multidisciplinary approach to treat a subgingivally fractured and intruded incisor - a case report.

[8]. James HS, Simon AB. Root extrusion- Rationale and techniques 1984;28:909-921

[9]. Tegsjo U, Valerius-Olsson H, Olgart K: Intra-alveolar transplantation of teeth with cervical root fractures. Swed Den J 1978;2:7382

[10]. Karin Z, Daniel Z, Aubrey S Doron H. Orthodontic forced eruption: case report of an alternative treatment of subgingivally fractured young permanent incisors. QuintessInt 1992;23:393-399. 